\title{
Efeito do tempo de conservação dos espécimes sobre a qualidade dos microincrementos em otólitos sagittae de Anchoa tricolor (Agassiz) (Clupeiformes, Engraulidae)
}

\author{
Joaquim N. de S. Santos ${ }^{1}$, Marcio de A. Silva ${ }^{1}$, Ruan M. Vasconcellos ${ }^{1}$ \& Francisco G. Araújo ${ }^{1,2}$ \\ 1 Universidade Federal Rural do Rio de Janeiro, Laboratório de Ecologia de Peixes, Antiga Rodovia Rio-São Paulo, Km 47, \\ 23851-970 Seropédica, Rio de Janeiro, Brasil.E-mail: jnssantos@yahoo.com.br \\ ${ }^{2}$ Autor correspondente: E-mail: gerson@ufrrj.br
}

\begin{abstract}
Effect of the conservation period of the specimens on the microstructure quality of sagittae otoliths in Anchoa tricolor (Agassiz) (Clupeiformes, Engraulidae). We compared the number of microstructure of Anchoa tricolor (Agassiz, 1829) post-larvae sagittae otoliths in specimens fixed in formalin $10 \%$ in different periods of conservation (four days versus four years). Significant differences were found in the number of microstructure for post-larvae ranging from $20-26 \mathrm{~mm}$ total length (TL), with averages of 18.6 microstructures ( \pm 0.75 standard error) for fishes preserved for four years, and 35.3 microstructures ( \pm 1.28 standard error) for fishes preserved for four days. Therefore, the number of post-larvae otoliths microstructure preserved for long periods can not be suitable to infer age or growth rates. Additionally, we failed in detecting and extracting otoliths in post-larvae smaller than $20 \mathrm{~mm}$ TL preserved due to its comparatively higher organic matter composition, mainly during the early life cycle.
\end{abstract}

KEY WORDS. Age, anchovy, post-larvae, conservation.

RESUMO. Efeito do tempo de conservação dos espécimes sobre a qualidade dos microincrementos em otólitos sagittae de Anchoa tricolor (Agassiz) (Clupeiformes, Engraulidae). O número de microincrementos em otólitos sagittae de espécimes de pós-larvas de Anchoa tricolor (Agassiz, 1829) fixadas em formol 10\% foram comparados em diferentes períodos de conservação (quatro dias versus quatro anos). Diferenças significativas foram encontradas no número de microincrementos para pós-larvas variando de $20-26 \mathrm{~mm}$ de comprimento total (CT), com médias de 18,6 microincrementos ( \pm 0.75 erro padrão) para peixes preservados por quatro anos, e 35,3 microincrementos $( \pm 1.28$ erro padrão) para peixes preservados por quatro dias. Portanto, o número de microincrementos em otólitos de póslarvas preservados por longos períodos pode não ser adequado para inferências de taxas de idade ou crescimento. Adicionalmente, não foram detectados ou extraídos otólitos em pós-larvas menores do que $20 \mathrm{~mm} C T$ devido a maior composição de matéria orgânica durante esta fase do ciclo de vida.

PALAVRAS CHAVE. Idade, manjubas, pós-larvas, conservação.

Estudos de crescimento de peixes utilizando otólitos são freqüentemente realizados em indivíduos fixados em formol e conservados em álcool. Dependendo do tempo que o material vai ser examinado, é essencial ter um conhecimento sobre os efeitos que os líquidos conservantes podem produzir sobre estas estruturas.

Otólitos são concreções resistentes de tamanho e composição química variáveis localizadas na cápsula ótica dos peixes actinopterígios, constituídas por uma matriz protéica mineralizada e carbonato de cálcio. A percentagem de material orgânico total presente nessas estruturas varia de 0,01 a 10\% nas diferentes espécies, sendo as maiores concentrações encon- tradas nos indivíduos mais jovens (DEgEns et al. 1969). TAKAGI et al. (2000) sugerem que uma matriz de carboidrato, produzida pelas células do epitélio sacular, é secretada na endolinfa e depositada no otólito, contribuindo para a constituição de glicoproteínas dessa estrutura.

A diminuição no tamanho e peso dos indivíduos, bem como dos otólitos, devido à ação dos líquidos conservantes, tem sido reportada freqüentemente na literatura (e.g. MCGURK 1985, Fox 1996, KRISTOFfersen \& SALVAnes 1998). Fatores de correção, para as variações na forma e tamanho, foram propostas por DARIUSz (2002) para sardinhas Clupea harengus (Linnaeus, 1758) e smelt Osmerus eperlamus (Linnaeus, 1758). No entanto, os danos que 

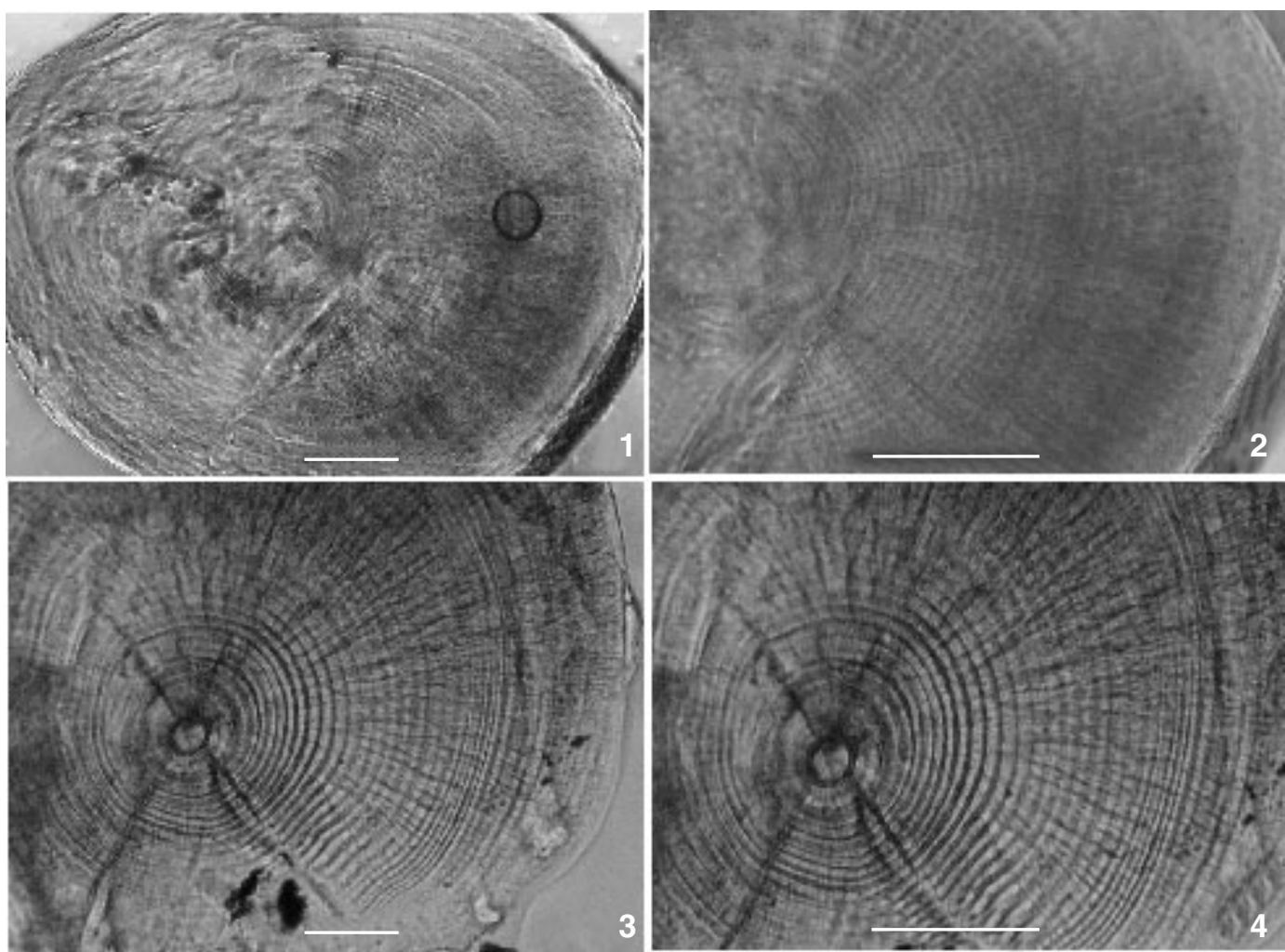

Figuras 1-4. Fotomicrografia dos otólitos conservados por quatro anos (1-2) e conservados por quatro dias (3-4). Barra de escala = $0,05 \mathrm{~mm}$. Observação: o círculo presente em $a$ é uma bolha de ar que se formou durante a preparação da lâmina.

ocorrem nos otólitos são, na maioria das vezes, irreparáveis ou fonte de erros na interpretação de dados Fox (1996). Os peixes, principalmente os juvenis, conservados em formol, podem ter seus otólitos desgastados parcialmente ou quebrados BROTHERs (1987), tornando-se inviável o estudo dos mesmos. O álcool, conservante mais comum dos peixes em coleções, quando diluído abaixo de $85 \%$ comporta-se como ácido fraco e pode danificar os otólitos de larvas (RADTKE \& WARWood 1980).

Nos últimos anos, vem ocorrendo um avanço significativo na implementação de métodos que permitem a determinação do crescimento de larvas de Engraulidae, com uma ampla aceitação de trabalhos que confirmam a periodicidade diária de microincrementos em otólitos (TAKAHASHI et al. 2001, CERMEÑO et al. 2003). Todavia, a contagem de microincrementos constitui quase sempre em uma fonte de erros, seja pela inexperiência dos leitores ou pela metodologia na extração e preparo dos otólitos (CAMPANa 2001). O objetivo do presente trabalho foi identificar a formação de microincrementos em otólitos sagittae de Anchoa tricolor (Agassiz, 1829), uma espécie em que os indivíduos apresentam curta longevidade e relevante importância comercial.

Foram utilizadas 30 pós-larvas medindo de 15 a $26 \mathrm{~mm}$ de comprimento total (CT), porém só foi possível a observação dos otólitos nos indivíduos acima de $20 \mathrm{~mm}$ CT. A extração da estru- tura foi feita com o auxílio de estiletes sob microscópio estereoscópio, obtendo-se um total de 20 otólitos sagittae: 10 de indivíduos que foram fixados em formol a $10 \%$ e transferidos para álcool 70\%, há cerca de quatro anos (2000); e outros 10 de indivíduos fixados somente em formol a 10\% por apenas quatro dias. Estes dois períodos de tempo (quatro dias e quatro anos) foram escolhidos para as comparações devido à disponibilidade de material em número suficiente. Foi feita a montagem em lâmina e lamínula, sem uma preparação prévia dos mesmos (não foram desgastados para a visualização dos microincrementos), como é usual para os otólitos da maioria das espécies de peixes de maior porte. A contagem dos microincrementos foi feita sob microscópio óptico, com as estruturas submetidas a aumentos de 200x e 400x, sendo fotografados com câmera digital de 3,1 milhões de pixels. Foram realizadas leituras por duas pessoas diferentes, além de uma terceira quando as contagens não coincidiam, até se chegar a uma concordância.

Foi observada a formação de microincrementos em otólitos, porém não foi determinada a periodicidade de suas formações. Indivíduos abaixo de $20 \mathrm{~mm} \mathrm{CT}$, conservados por cerca de quatro anos, não apresentaram otólitos passíveis de detecção e extração através da inspeção da cápsula auditiva, sugerindo que os mesmos foram desgastados ou fragmentados

Revista Brasileira de Zoologia 22 (4): 949-952, dezembro 2005 

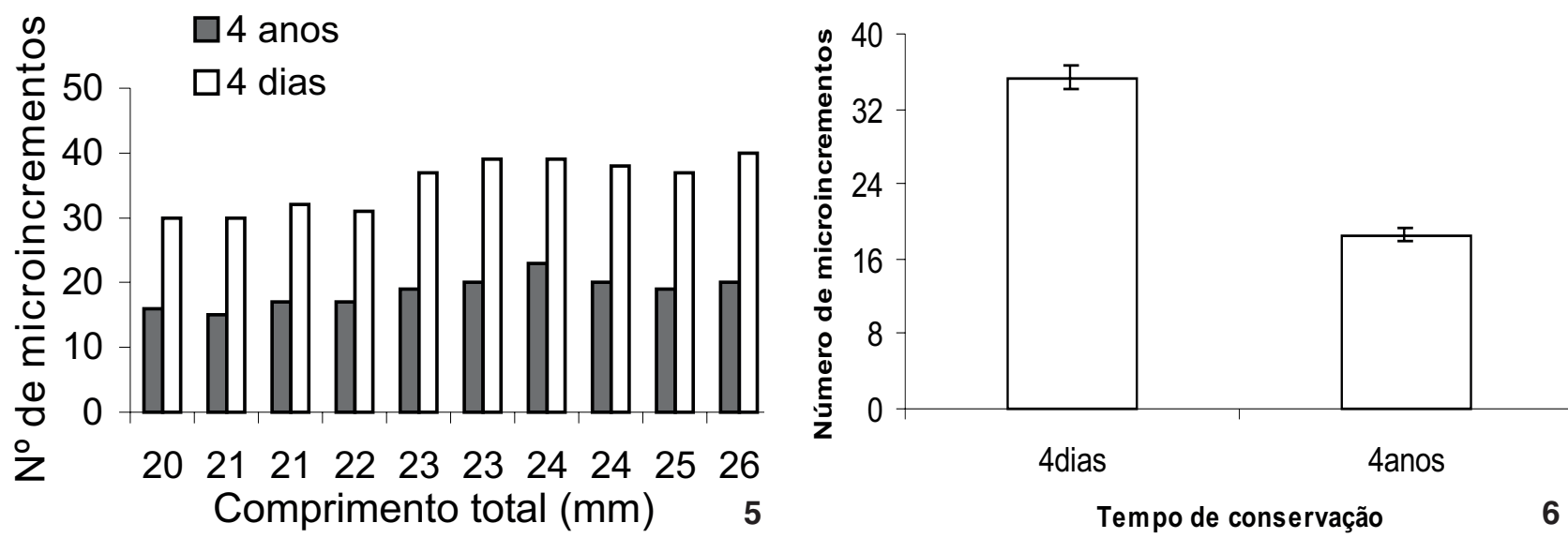

Figuras 5-6. Número (5) e número médio ( \pm erro padrão) (6), de microincrementos em otólitos sagittae de pós-larvas de $A$. tricolor em diferentes tempos de conservação.

pela ação do formol ou do álcool. Em pós-larvas de maior tamanho (CT > 20mm) isto não ocorreu, sendo possível a localização e extração dos otólitos, porém não apresentaram boa qualidade para a visualização de todos os micro-incrementos presentes (Figs 1-4). Por outro lado, aqueles extraídos de póslarvas conservadas por quatro dias apresentaram microincrementos de boa qualidade e fácil visualização, proporcionando uma leitura mais consistente e íntegra dos mesmos.

A média de microincrementos para indivíduos conservados por quatro dias foi de 35,30 (erro padrão $=1,28$ ) tendo sido significativamente maior $(\mathrm{p}<0,01)$ em relação aos indivíduos conservados por quatro anos, que apresentaram média de 18,60 microincrementos (erro padrão $=0,75$ ) (Figs 5 e 6). Esta diferenciação no número de microincrementos corrobora a hipótese de que a qualidade e a nitidez dos mesmos são influenciadas pelo tempo de exposição aos líquidos conservantes, conseqüentemente os trabalhos baseados em material conservado por longos períodos tendem a subestimar o número de microincrementos. KRISTOFFERSEN \& SALVANES (1998), verificaram desgastes significativos em otólitos de Benthosema glaciale (Reinhardt, 1837), uma espécie mesopelágica também de pequeno porte, os quais foram conservados durante 200 dias em formol e álcool, cujos desgastes foram proporcionais ao tempo de exposição.

A mais baixa qualidade dos microincrementos observados nos otólitos conservados por longo período foi possivelmente causada pelo desgaste ou "digestão" da parte orgânica, que, em formas juvenis, pode estar presente em maiores quantidades. A causa da não detecção de otólitos em pós-larvas com tamanhos menores de $20 \mathrm{~mm}$ CT também pode ter sido devido à "digestão" dos mesmos por razão semelhante.

\section{AGRADECIMENTOS}

Ao professor Armando Sales da área de Histologia Animal, UFRRJ, pelo apoio de infra-estrutura laboratorial, e ao amigo Benjamin C. T. Pinto, pelas sugestões no decorrer do trabalho. Este trabalho foi realizado com apoio da CAPES, através da concessão de bolsa de mestrado ao primeiro autor.

\section{REFERÊNCIAS BIBLIOGRÁFICAS}

Brothers, E.B. 1987. Methodological approaches to the examination of otoliths in aging studies, p. 319-330. In: R.C. Summerfelt \& G.E. Hall (Eds). Age and growth of fish. Ames, Iowa State University Press, 544p.

CAMPANA, S.E. 2001. Accuracy, precision and quality control in age determination, including a review of the use and abuse of age validation methods. Journal of Fish Biology, Dunscore, 59: 197-242.

Cermeño, P.; Uriarte, A.; Murguia; A.M. \& B. Morales-Nin. 2003. Validation of daily increment formation in otoliths of juvenile and European anchovy. Journal of Fish Biology, Dunscore, 62: 679-691.

DARIUSz, P.F. 2002. Length correction of larval and early-juvenile herring (Clupea harengus L.) and smelt (Osmerus eperlanus L.) after preservation in formalin and alcohol. Bulletin of the Sea Fisheries Institute, Gdynia, 1 (155): 47-51.

Degens, E.T.; W.G. Deuser \& R.L. Haerdrich 1969. Molecular structure and composition of fish otoliths. Marine Biology, Luhe, 2: 105-113.

Fox, C.J. 1996. Length changes in herring Clupea harengus larvae: effects of capture and storage in formaldehyde and alcohol. Journal of Plankton Research, Oxford, 18: 483-493.

Kristoffersen, J.B \& A.G.V. SALVANES. 1998. Effects of formaldehyde and ethanol preservation on body and otoliths of Maurolicus muelleri and Benthosema glaciale. Sarsia, Bergen, 83: 95-102.

MCGURK, M.D. 1985. Effects of net capture on the post preservation morphometric, dry weigth, and condition factor of pacific herring larvae. Transaction of the American Fisheries Society, Bethesda, 114 (3): 348-355.

RADTKE, R.L. \& K.G. WaIWOOD. 1980. Otolith formation and body 
shrinkage due to fixation in larval cod Gadus morhua. Canadian Technical Report of Fisheries and Aquatic Sciences, Québec, 929: 1-10.

Takahashi, M.; Y. Watanabe; T. Kinoshita \& C. Watanabe. 2001. Growth of larval and early juvenile Japanese anchovy,

Recebido em 01.IV.2005; aceito em 20.X.2005
Engraulis japonicus, in the Kuroshio-Oyashio transition region. Fisheries Oceanography, La Jolla, 10: 235-247.

TAKagi, Y.; K. Ishida \& Y. Mugrya. 2000. Carbohydrates of the otolith organ in the rainbow trout Oncorhynchus mykiss detected by lectins. Fisheries Science, Minato, 66: 933-939. 